\title{
SARS-CoV-2 / COVID-19 in patients on the Swiss national transplant waiting list
} \author{
Déla $^{d}$, Hadaya Karine ${ }^{f}$, Mueller Thomas F. ${ }^{g}$, Parvex Paloma ${ }^{f}$, Immer Franz F. ${ }^{a}$ \\ a Swisstransplant, Berne, Switzerland \\ b University of Zurich Faculty of Medicine, Zurich, Switzerland \\ c Epidemiology, Biostatistics and Prevention Institute, University of Zurich, Switzerland \\ d Lausanne University Hospital, Lausanne, Switzerland \\ e Cantonal Hospital St Gallen, Switzerland \\ f Geneva University Hospitals, Geneva, Switzerland \\ g University Hospital Zurich, Switzerland
}

Benden Christian $^{\text {ab }}$, Haile Sarah R. ${ }^{c}$, Kruegel Nathalie ${ }^{a}$, Beyeler Franziska ${ }^{a}$, Aubert John-David ${ }^{d}$, Binet Isabelle ${ }^{e}$, Golshayan

\section{Summary}

AIMS OF THE STUDY: The impact of coronavirus disease 2019 (COVID-19) on patients listed for solid organ transplantation has not been systematically investigated to date. Thus, we assessed occurrence and effects of infections with severe acute respiratory syndrome coronavirus 2 (SARS-CoV-2) on patients on the Swiss national waiting list for solid organ transplantation.

METHODS: Patient data were retrospectively extracted from the Swiss Organ Allocation System (SOAS). From 16 March to 31 May 2020, we included all patients listed for solid organ transplantation on the Swiss national waiting list who were tested positive for SARS-CoV-2. Severity of COVID-19 was categorised as follows: stage I, mild symptoms; stage II, moderate to severe symptoms; stage III, critical symptoms; stage IV, death. We compared the incidence rate (laboratory-confirmed cases of SARS-CoV-2), the hospital admission rate (number of admissions of SARS-CoV-2-positive individuals), and the case fatality rate (number of deaths of SARS-CoV-2-positive individuals) in our study population with the general Swiss population during the study period, calculating age-adjusted standardised incidence ratios and standardised mortality ratios, with $95 \%$ confidence intervals $(\mathrm{Cls})$.

RESULTS: A total of 1439 patients were registered on the Swiss national solid organ transplantation waiting list on 31 May 31 2020. Twenty-four (1.7\%) waiting list patients were reported to test positive for SARS-CoV-2 in the study period. The median age was 56 years (interquartile range 45.3-65.8), and 14 (58\%) were male. Of all patients tested positive for SARS-CoV-2, two patients were asymptomatic, 14 (58\%) presented in COVID-19 stage I, $3(13 \%)$ in stage II, and $5(21 \%)$ in stage III. Eight patients (33\%) were admitted to hospital, four $(17 \%)$ required intensive care, and three (13\%) mechanical ventilation. Twenty-two patients $(92 \%)$ of all those infected recovered, but two male patients aged $>65$ years with multiple comorbidities died in hospital from respiratory failure. Comparing our study population with the general Swiss population, the age-adjusted standardised incidence ratio was 4.1 (95\% $\mathrm{Cl}$ 2.7-6.0).

CONCLUSION: The overall rate of SARS-CoV-2 infections in candidates awaiting solid organ transplantation was four times higher than in the Swiss general population; however, the frequency of testing likely played a role. Given the small sample size of affected patients, conclusions have to be drawn cautiously and results need verification in larger cohorts

Keywords: COVID-19, organ transplantation, SARSCoV-2, waiting list

\section{Introduction}

At the end of 2019, a novel coronavirus, severe acute respiratory syndrome coronavirus 2 (SARS-CoV-2), emerged in the Chinese province of Hubei, causing a pandemic of unknown extent $[1,2]$. The disease caused by SARS-CoV-2 was named coronavirus disease 2019 (COVID-19) by the World Health Organization and declared an international public health emergency on 30 January 2020 [2]. The COVID-19 pandemic has an ongoing tremendous impact across the world with major consequences in health care, world economy, and social affairs. As vaccinations have just commenced and effective therapy options are limited, the number of new cases and the death toll are continuously rising [2]. In Europe, Italy was the first country severely hit by the pandemic [3]. On a daily basis, thousands of northern Italian citizens cross the Italian-Swiss border to the canton of Ticino, the southernmost canton of Switzerland, to commute to work; thus, it was expected that Switzerland would face a similar healthcare crisis as Italy. On 24 February 2020, the first SARS-CoV-2 positive individual was diagnosed in Switzerland (canton of Ticino) according to Swiss Federal Office of Public Health (FOPH) [4]. Initially, the impact of the pandemic on healthcare systems in China and Italy emerged, in par- 
ticular, shortness of intensive care unit (ICU) capacity and lack of hospital resources; in addition, there was primarily scarce information on COVID-19 and its effect on organ donation and solid organ transplantation [5].

Like Italy, where particularly Lombardy in the north of the country was struck by the pandemic, the pandemic also grew heterogeneously in Switzerland with its 8,700,000 citizens, affecting the various cantons differently. The highest incidence of SARS-CoV-2 infection was recorded in the French- and Italian-speaking Swiss cantons, with $>100$ confirmed cases per 10,000 inhabitants in the canton of Geneva and $>90$ confirmed cases per 10,000 inhabitants in the canton of Ticino [6].

Early on in the pandemic, Loupy and coworkers published data to quantify the impact of the COVID-19 pandemic on organ donation and transplantation in France and the USA, showing a clear association between the rise of COVID-19 infections and the fall in numbers of solid organ transplantation [7]. There was an over $90 \%$ overall drop in deceased organ transplant procedures since the start of the pandemic in France and over 50\% in the USA [7]. The decline in transplant activities has consequences for the vulnerable population of patients with end-stage organ disease waiting for solid organ transplantation. Sharma and colleagues from the UK showed that an estimated 1670 kidney transplant opportunities potentially were gone as a result of the pandemic in the UK, leading to an almost $50 \%$ increase in patients awaiting kidney transplant based on usual transplant figures [8]. The latter is very concerning given the fact that patients on dialysis awaiting kidney transplant are a vulnerable sub-group of patients, frequently with multiple comorbidities. A recent meta-analysis including more than 70,000 patients from 21 studies revealed that cardiovascular complications in particular are common in patients diagnosed with COVID-19, likely adding to COVID-19-associated morbidity and mortality [9]. However, there is a paucity of data on SARS-CoV-2 infection and COVID-19 in patients with end-stage organ failure awaiting solid organ transplantation. A recent publication of (lately published) first experiences of SARS-CoV-2 in solid organ transplantation in Switzerland focused only on patients post-transplant (after solid organ transplantation) using Swiss Transplant Cohort Study (STCS) data [10]. The study by Tschopp et al. revealed that clinical symptoms of COVID-19 in middle-aged solid organ transplantation patients appeared comparable to the general population, without higher complication rates detected [10].

To our knowledge, SARS-CoV-2 infection rates and disease outcomes of COVID-19 in a well-defined group of patients with end-stage organ failure listed for solid organ transplantation have not been investigated to date. Thus, we aimed to assess SARS-CoV-2 infection rates and COVID-19 and its disease-related risks and outcomes in patients on the Swiss national solid organ transplantation waiting list. We hypothesised that the overall rate of SARS-CoV-2 infections in solid organ transplantation candidates is probably comparable to that in the general population, but that the morbidity and mortality rate of COVID-19 is likely to be raised.

\section{Materials and methods}

\section{Study population}

For the purpose of our study, patient data were retrospectively extracted from the Swiss Organ Allocation System (SOAS). During the study period from 16 March to 31 May 31 2020, we included all adult and paediatric patients $(<18$ years of age) listed for solid organ transplantation on the Swiss national waiting list who were tested positive for SARS-CoV-2, mandatorily reported to Swisstransplant by their transplant centre and subsequently paused until clinical recovery and exclusion of ongoing SARS-CoV-2 infection by a negative test result. However, there was no nationwide policy on systematic serial testing for SARSCoV-2 of patients on the national solid organ transplantation waiting list throughout the entire study period. The patients' follow-up terminated at the end of the observation period on 31 May 2020.

We collected patients' characteristics including sex, age, transplant organ type, waiting list status (active vs inactive), waiting list times, smoking status and comorbidities. With regards to the circumstances at time of first diagnosis of SARS-CoV-2 infection, we compiled data on laboratory values, chest imaging, clinical course (hospital admission; ICU admission; mechanical ventilation), COVID-19-associated clinical complications, and clinical outcomes (full recovery; recovered with sequelae; not yet recovered; death). Severity of COVID-19 was subcategorised using the criteria of Siddiqi and Mehra: stage I, mild symptoms (e.g., dry cough, fatigue, headache); stage II, moderate to severe symptoms (e.g., dyspnoea, hypoxia); stage III, critical symptoms (e.g., acute respiratory distress syndrome [ARDS], cardiac failure); stage IV, death [11].

To accomplish our project, we used data collected by Swisstransplant and stored in the SOAS for quality control purposes amongst others, as mandated by the Swiss Federal Office of Public Health (FOPH). Our research was conducted in accordance with the Helsinki Declaration as revised in 2013.

\section{Statistical analysis}

Descriptive statistics were utilised to describe patients' demographics, COVID-19 stage and clinical outcome. Data were summarised as $\mathrm{n}(\%)$ or median (interquartile range, IQR). We compared the incidence rate (laboratory-confirmed cases of SARS-CoV-2), the hospital admission rate (number of admissions of SARS-CoV-2-positive individuals) and the case-fatality rate (number of deaths of SARSCoV-2-positive individuals) in our study population with the general population of Switzerland (and the Principality of Liechtenstein) during the study period, calculating ageadjusted standardised incidence ratios (SIRs) and standardised mortality ratios (SMRs), and 95\% confidence intervals (CIs) [12]. Publicly available data of the FOPH were used [13-15].

\section{Results}

At the end of the study period on 31 May 2020, a total of 1439 patients were registered on the Swiss national solid organ transplantation waiting list in the SOAS. In detail, 1027 (71\%) of patients awaited kidney transplant, $211(15 \%)$ awaited liver transplants, $91(6 \%)$ heart trans- 
plants, $47(3 \%)$ lung transplants, and $63(4 \%)$ other transplant types (pancreas, small bowel, and multi-organ transplants). Only $1 \%$ of waiting list patients $(n=15)$ belonged to the paediatric age group $(<18$ years of age). Of the total 1439 waiting list patients, $744(52 \%)$ were actively listed for transplant on 31 May 2020, 695 (48\%) were temporarily paused on the waiting list, most commonly in the sub-group of patients awaiting kidney transplant $(\mathrm{n}=520$; $75 \%$ ).

In the observation period from 16 March to 31 May 2020, a total of $24(1.7 \%)$ national waiting list patients (including 1 paediatric patient aged $<18$ years of age) were reported to test positive for SARS-CoV-2 RNA; all these patients were reported to Swisstransplant by the centre according to Swiss national recommendations and subsequently temporarily paused on the waiting list to monitor SARS-CoV-2 infection, clinical development and outcomes cases of COVID-19. The median age of the SARS-CoV-2-infected patients was 56 years (IQR 45.6-65.8), 10 patients (42\%) were 60 years of age or older, and more than half of patients were male $(14 ; 58 \%)$. The vast majority of patients awaited kidney transplant $(\mathrm{n}=21 ; 88 \%)$, all of them on renal replacement therapy, of the remaining three patients, two awaited abdominal double organ transplantation, and one patient lung transplant. With regards to the total number of patients on the waiting list according to primary indication for solid organ transplantation, $2 \%$ of patients on the kidney transplant waiting list and $2 \%$ of patients on the lung transplantation waiting list were infected. At the time of SARS-CoV-2 diagnosis, 14 (58\%) were actively listed for solid organ transplantation; this figure was similar in the largest sub-group of patients awaiting kidney transplant. Median waiting list time was 946 days (IQR 437-1449) for the whole group of 24 patients, and the median waiting list time for the sub-group of patients awaiting kidney transplant was 982 days (IQR 582-1521). The median follow-up time of the study cohort was 53 days (IQR 34-58).

As to be expected in a group of patients awaiting predominantly kidney transplant, comorbidities were very frequent. Eighty-one percent of kidney transplant waiting list patients suffered from hypertension, and it was the most common comorbidity also in the overall patient population $(\mathrm{n}=24,79 \%)$ patients. The second most frequent comorbidity was obesity in $42 \%$ of the overall patient cohort and $43 \%$ in the sub-group of patients awaiting kidney transplant. Further details on comorbidities are displayed in table 1. Psychiatric or neurological complications associated with COVID-19 were not investigated routinely as part of this study. One kidney transplant candidate aged $>65$ years was an active smoker; data on smoking status was available in $23(96 \%)$ patients.

Only two (8\%) of the patients tested positive for SARSCoV-2 were asymptomatic. As shown in table 2, 14 (58\%) of the patients presented with COVID-19 stage I according to Siddiqi and Mehra [11], 3 (13\%) of patients stage II, and $5(21 \%)$ with stage III. For further details see table 2.

Laboratory values were available for only some of the patients, as detailed in table 3. Further, seven (44\%) of patients were leucopenic and two (18\%) were lymphopenic. The median C-reactive protein (CRP) value was mildly raised (16 mg/l, IQR 2.0-155; normal range in adults $<5$ $\mathrm{mg} / \mathrm{l}) ; 10(71 \%)$ patients presented with a CRP value above the normal range. The median procalcitonin level (available in $29 \%$ of patients) was moderately elevated $(1.8 \mu \mathrm{g} /$ 1, IQR 0.7-2.8); normal adult value $<0.5 \mu \mathrm{g} / \mathrm{l}$ ) with six $(86 \%)$ patients having an abnormal procalcitonin level. Chest imaging (imaging technique not further specified) was performed in $13(54 \%)$ of the patient cohort and diagnosed as abnormal in $6(46 \%)$ of those cases.

With regards to the clinical course following COVID-19 diagnosis, eight patients (33\%) were admitted to the hospital, four $(17 \%)$ were admitted to the ICU, and three $(13 \%)$ required mechanical ventilation (details for the sub-group of kidney transplant candidates see table 3). The most frequent complications among study patients were pneumonia and ARDS, both occurred only in patients awaiting kidney transplant (table 3 ).

Of the overall patient cohort, $22(92 \%)$ recovered following SARS-CoV-2 infection, and $100 \%$ of the non-kidney transplant candidates recovered. Unfortunately, two patients $(8 \%)$ died during the observation period due to COVID-19. Both patients were male, aged $>65$ years, awaiting kidney transplant, and each suffered from more than three comorbidities. Both patients needed hospital and

Table 1: Comorbidities of patients on the waiting list tested positive for SARS-CoV-2.

\begin{tabular}{|c|c|c|c|}
\hline Comorbidities & $\begin{array}{c}\text { All } \\
n=24(100)\end{array}$ & $\begin{array}{c}\mathrm{KTX} \\
\mathrm{n}=\mathbf{2 1}(88)\end{array}$ & $\begin{array}{c}\text { Other } \\
n=3(12)\end{array}$ \\
\hline Chronic heart failure & $1(4)$ & $1(5)$ & - \\
\hline Chronic lung disease & $2(8)$ & $2(10)$ & - \\
\hline Diabetes mellitus & $4(17)$ & $3(14)$ & $1(33)^{*}$ \\
\hline Hypertension & $19(79)$ & $17(81)$ & $2(67)$ \\
\hline Obesity & $10(42)$ & $9(43)$ & $1(33)$ \\
\hline Peripheral artery disease & $4(17)$ & $4(19)$ & - \\
\hline
\end{tabular}

KTX $=$ kidney transplantation. Data are presented as number $(\%) .{ }^{*} \mathrm{n}=1$ paediatric patient.

Table 2: COVID-19 stages at time of positive testing for SARS-CoV-2.

\begin{tabular}{|l|c|c|}
\hline & $\begin{array}{c}\text { All } \\
\mathbf{n = 2 4 ( 1 0 0 )}\end{array}$ & $\begin{array}{c}\text { Other } \\
\mathbf{n = 3}(\mathbf{1 2})\end{array}$ \\
\hline COVID-19 Stage I* & $14(58)$ & $13(62)$ \\
\hline COVID-19 Stage II* $^{*}$ & $3(13)$ & $3(14)$ \\
\hline COVID-19 Stage III $^{*}$ & $5(21)$ & - \\
\hline
\end{tabular}

COVID-19 = coronavirus disease 2019; SARS-CoV-2 = severe acute respiratory syndrome coronavirus 2; KTX = kidney transplantation. Data are presented as number (\%). * COVID-19 stages were adapted from Siddiqi and Mehra [10]. 
ICU admission and mechanical ventilation as they developed pneumonia followed by ARDS. None of the study patients underwent transplantation during the study period.

In the comparison our study population with the general population of Switzerland and the Principality of Liechtenstein (8.74 million) $[13,14]$, there were a total of 30,924 laboratory-confirmed cases of SARS-CoV-2 in the study period [15], resulting in a SIR of $4.1(95 \%$ CI 2.7-6.0). Further, a total of 3972 SARS-CoV-2 positive individuals were admitted to hospital in Switzerland and the Principality of Liechtenstein in the study period [15], resulting in an age-adjusted SIR for hospital admission of 10.5 (95\% CI 5.3-21.1). Of the 30,838 laboratory-confirmed cases of SARS-CoV-2 in the general population, 1657 individuals died due to COVID-19, resulting in an age-adjusted SMR of 14.2 (95\% CI 3.6-57.0).

\section{Discussion}

Our study investigating SARS-CoV2 infection rates and COVID-19 outcomes in candidates on the national Swiss waiting list for solid organ transplantation revealed that only a minority of $<2 \%$ of a total of over 1400 registered waiting list patients tested positive for SARS-CoV-2 between 16 March and 31 May 2020, 21 (88\%) of those candidates for kidney transplant. Over half of patients $(\mathrm{n}=$ $14,58 \%$ ) presented with mild disease (COVID-19 stage I), and 5 (21\%) with advanced disease (COVID-19 stage III). Yet overall post-COVID-19 outcome was favourable, with 9 out of 10 patients recovering, 2 patients (8\%) passed away due to COVID-19-associated complications. In contrast, Boyarsky et al. from Baltimore, USA, revealed a higher positive rate of diagnostic SARS-CoV-2 tests of $17 \%$ among 152 patients with end-stage renal disease [16]. The overall rate of SARS-CoV-2 infections in our study population was four times higher than in the general population, although the frequency of testing likely plays a role here. The SARS-CoV-2 positive transplant candidates were significantly more often hospitalised. Furthermore, our SARS-CoV-2-positive study patients died more often from COVID-19 compared with the general Swiss population.

To the authors' knowledge, there is a lack of published data regarding SARS-CoV-2 infection and COVID-19 in patients on a solid organ transplantation waiting list during the pandemic. Published literature to date reporting on the COVID-19 pandemic and solid organ transplantation focusses primarily on the pandemic's effect on organ donation itself and transplant activity, rather than on SARSCoV-2 infection rates and COVID-19 in candidates awaiting solid organ transplantation. The COVID-19 pandemic struck Switzerland heterogeneously, with the highest incidence of SARS-CoV-2 infection recorded in the French- and Italian speaking cantons neighbouring France and Italy, respectively [6].

In our study, $88 \%$ of the patients tested positive for SARS$\mathrm{CoV}-2$ during the study period were awaiting kidney transplant requiring dialysis. As all of the kidney transplant waiting list patients underwent renal replacement therapy, the frequency of SARS-CoV-2 testing might play a role. Furthermore, the two deaths in the study population occurred in patients awaiting kidney transplant with multiple comorbidities including hypertension and obesity. Hernández-Galdamez et al. from Mexico investigated the increased risks of hospitalisation and death in patients with COVID-19 and pre-existing noncommunicable diseases [17]. The cross-sectional national study including more than 210,000 confirmed COVID-10 cases officially reported to the Mexican health authorities up to 27 June 2020 showed that almost $50 \%$ of patients with COVID-19 suffered from a comorbidity, the most common being hypertension in one out of five cases [17]. Chronic kidney disease raised the risk of death the most (odds ratio [OR] 2.31), followed by diabetes (OR 1.69), immunosuppression (OR 1.62), obesity (OR 1.42), hypertension (OR 1.24) and chronic obstructive pulmonary disease (OR 1.20) [13]. Comorbidities augmenting ICU admission risk and intubation risk were diabetes, immunosuppression and obesity [17]. In our study population, hypertension and obesity were the most frequent comorbidities, in $19(79 \%)$ and $10(42 \%)$ of patients, respectively. Further, four (17\%) of patients were diagnosed with diabetes. In comparison, all of these are less common in the Swiss general population; $17.6 \%$ of the general population in Switzerland suffers with hypertension according to the Swiss Health Observatory in $2017 ; 11.3 \%$ of the Swiss general population

Table 3: COVID-19 clinical diagnostics and outcome

\begin{tabular}{|c|c|c|c|}
\hline Variables & $\begin{array}{c}\text { All } \\
n=24(100)\end{array}$ & $\begin{array}{c}\mathrm{KTX} \\
\mathrm{n}=\mathbf{2 1}(88)\end{array}$ & $\begin{array}{c}\text { Other } \\
n=3(12)\end{array}$ \\
\hline \multicolumn{4}{|l|}{ Laboratory values, median (IQR) } \\
\hline Leucocyte count $(\mathrm{G} / \mathrm{l}), \mathrm{n}=16$ & $5.0(3.1-6.8)^{*}$ & & \\
\hline Lymphocyte count (G/l),n = 11 & $0.8(0.4-1.7)^{*}$ & & \\
\hline $\mathrm{CRP}(\mathrm{mg} / \mathrm{l}), \mathrm{n}=14$ & $16(2.0-155)^{*}$ & & \\
\hline Procalcitonin $(\mu \mathrm{g} / \mathrm{l}), \mathrm{n}=7$ & $1.8(0.7-2.8)^{*}$ & & \\
\hline \multicolumn{4}{|l|}{ Clinical course } \\
\hline Hospital admission & $8(33)^{*}$ & $7(33)$ & $1(33)$ \\
\hline ICU admission & $4(17)$ & $4(19)$ & - \\
\hline Mechanical ventilation & $3(13)$ & $2(15)$ & - \\
\hline \multicolumn{4}{|l|}{ Complications } \\
\hline ARDS & $5(21)$ & $5(24)$ & - \\
\hline Pneumonia & $8(33)$ & $8(38)$ & - \\
\hline Acute kidney injury & - & - & - \\
\hline Acute thromboembolic event & $1(4)$ & $1(5)$ & - \\
\hline
\end{tabular}

ARDS = acute respiratory distress syndrome; COVID-19 = coronavirus disease 2019; CRP = C-reactive protein; ICU = intensive care unit; IQR = interquartile range; KTX = kidney transplantation Data are presented as numbers (percent) unless indicated otherwise. ${ }^{*} n=1$ paediatric patient 
was obese and $4 \%$ of the general population was affected by diabetes in 2017 [18].

Alp Ikeler from Nashville, USA, highlighted in a recently published editorial the higher SARS-CoV-2 transmission risk among dialysis patients due to logistical issues of delivering kidney replacement therapy [19]. Patients with end-stage renal disease on dialysis require regular (day care) admissions to the dialysis unit, usually multiple times per week, and patients are kept longer in proximity with fellow patients also undergoing dialysis [19]. Therefore, it is imperative to put the appropriate strategies in place to prevent SARS-CoV-2 transmission in dialysis units [19]. To the authors' knowledge, SARS-CoV-2 cross infection at dialysis units in Switzerland was not reported during the study period.

Another important aspect is the known older age of dialysis patients with regards to COVID-19 [19]. Poletti et al. investigated age-specific SARS-CoV-2 infection fatality ratio and associated risk factors during the COVID-19 pandemic in Italy [20]. Based on the analysis of more than 5000 close contacts of COVID-19 cases, the infection fatality ratio was $0.5 \%$ for people $<70$ years of age and $10.5 \%$ for older people. Further, risk of death after SARSCoV-2 infection turned out to be 1.8 -fold higher for males [20]. In our study population, the only two fatalities were over 65 years old and male. Compared with the general population, our study population was older $(42 \%$ of transplant candidates were $\geq 60$ years of age vs $25 \%$ in the general population), and $58 \%$ of our study population were male compared to $49 \%$ in the general population [13, 14].

In our study, laboratory values at COVID-19 diagnosis were available in only a sub-group of study patients, owing to its retrospective design. The limited data set revealed a minimally reduced median lymphocyte count, a mildly raised median CRP, and a moderately elevated median procalcitonin level. A latest systematic review and metaanalysis evaluating clinical data, including laboratory findings, radiographic features and outcomes of $>60,000$ individuals diagnosed with COVID-19, demonstrated that CRP was raised in over three quarters of cases and lymphopenia present in almost two thirds of cases [21].

In order to ensure ongoing organ donation, protect solid organ transplantation candidates and maintain transplant activity even during the pandemic, international societies such as the Transplant Infectious Diseases Section (TID) of the Transplantation Society (TTS) had already set up consensus guidance on COVID-19 for transplant clinicians early in the pandemic, but the recommendation provided is almost completely based on international expert opinion only, with a low level of evidence [22-25]. The guidance documents are "work in progress" continuously updated on the basis of newer evidence emerging as the international expert community learns more about COVID-19 while the pandemic continues to heavily impact on health systems worldwide and cause fatalities. A group of experts from Texas provides guidance on solid organ transplantation candidates that were implemented in the TTS consensus guidance document [22]. All transplant candidates with active COVID-19 are prohibited from undergoing transplantation. Transplant candidates are to be assessed clinically and screened, mostly by nasopharyngeal or deep throat swabs, to rule out SARS-CoV-2 by polymerase chain-re- action testing (PCR); local protocols vary depending on the pandemic status of the area. During the pandemic in Switzerland, there was no general screening programme for all the patients on the national solid organ transplantation waiting list, but a negative SARS-CoV-2 screening test has been mandatory within 72 hours prior to organ allocation for both the transplant candidates and the donor in order to proceed with transplant. To date, no donorto-recipient SARS-CoV-2 transmission has occurred in Switzerland (Franz F Immer, personal communication).

Passamonti et al. have recently published their experience of solid organ transplantation in the COVID-19 pandemic, evaluating the COVID-19 incidence and mortality in all individuals undergoing solid organ transplantation during the pandemic in the North Italy Transplant Programme [26]. A total of 124 patients received solid organ transplantations in the study period, $5(4 \%)$ were diagnosed with COVID-19 (mean of 13 days post-solid organ transplantation). Five patients died during the study period; however, only one patient died from COVID-19 (COVID-19-associated mortality $0.8 \%$ ) [26].

\section{Strength and limitations}

It is the strength of our study that it investigated for the first time SARS-CoV-2 infection rates and COVID-19 outcomes in patients awaiting solid organ transplantation nationwide using a central data base. However, our study also has limitations, inherent to its nature as a registry data base study in a country with a current population of 8.7 million without systematic screening of all waiting list patients for SARS-CoV-2; thus, SARS-CoV-2 might be underdiagnosed, as patients with mild symptoms might not have had timely testing. Further, as a registry data base study, accuracy of data entry depends on the centres, in particular the extent of completeness of clinical data compiled at time of first diagnosis of SARS-CoV-2 infection, potentially creating bias. On the other hand, as all patients on the kidney transplant waiting list were on renal replacement therapy, testing for SARS-CoV-2 probably occurred more frequently in these patients. Laboratory values, for example, have to be interpreted carefully; no standardised laboratory protocol was used as real world data were collected, and our sample size was small. Likewise, data on chest imaging were not available for all patients, and if so, provided information only in the event of abnormal radiology. No specifics were available for the most common radiographic signs. In addition, no data were accessible on potential long-term sequelae of COVID-19 beyond the end of the observation period on 31 May 2020. Owing to data protection issues, we were also unable to investigate the regional impact of SARS-CoV-2 infection and COVID-19 on solid organ transplantation candidates, knowing that Swiss cantons were heterogeneously hit by the pandemic. Data on clinical stages of COVID in laboratory-confirmed cases of SARS-CoV-2 in the general population were not publicly available.

\section{Conclusion}

The overall rate of SARS-CoV-2 infections in candidates awaiting solid organ transplantation was four times higher than in the Swiss general population; however, frequency of testing likely played a role. SARS-CoV-2-positive wait- 
ing list patients were hospitalised much more often and more likely to die from COVID-19 compared with the general population in Switzerland. Preventive strategies remain generally important in the ongoing pandemic. In the future, vaccination should be given priority to population groups determined at risk based on published evidence.

\section{Acknowledgments}

The authors would like to thank all medical and allied health professional teams at the Swiss transplant centres for their invaluable collaborative work during the SARS-CoV-2 / COVID-19 pandemic. The authors also thank Andreas Elmer, MSc ETH, Research Associate at Swisstransplant, for his statistical support.

\section{Disclosure statement}

This study received no funding and no potential conflict of interest relevant to this article was reported.

\section{References}

1 Aslam S, Mehra MR. COVID-19: Yet another coronavirus challenge in transplantation. J Heart Lung Transplant. 2020;39(5):408-9. doi: http://dx.doi.org/10.1016/j.healun.2020.03.007. PubMed.

2 Hage R, Steinack C, Benden C, Schuurmans MM. COVID-19 in patients with solid organ transplantation: a systematic review. Transplantology. 2020;1(1):1-15. doi: http://dx.doi.org/10.3390/transplantology 1010001.

3 Distante C, Piscitelli P, Miani A. COVID-2019 outbreak progression in Italian regions: approaching the peak by the end of March in Northern Italy and first week of April in Southern Italy. Int J Environ Res Public Health. 2020;17(9):3025. doi: http://dx.doi.org/10.3390/ ijerph17093025. PubMed.

4 COVID-19 in Switzerland. 2020 February 24. [Online]. Accessed on: 2020 August 5. Available from: https://covid-19-schweiz.bagapps.ch/ de-2.html

5 Woolley AE, Mehra MR. Dilemma of organ donation in transplantation and the COVID-19 pandemic. J Heart Lung Transplant. 2020;39(5):410-1. doi: http://dx.doi.org/10.1016/j.healun.2020.03.017. PubMed.

6 COVID-19 information for Switzerland. 2020 May 29. [Online]. Accessed on: 2020 August 5. Available from: http://www.corona-data.ch

7 Loupy A, Aubert O, Reese PP, Bastien O, Bayer F, Jacquelinet C. Organ procurement and transplantation during the COVID-19 pandemic. Lancet. 2020;395(10237):e95-6. doi: http://dx.doi.org/10.1016/ S0140-6736(20)31040-0. PubMed.

8 Sharma V, Shaw A, Lowe M, Summers A, van Dellen D, Augustine T. The impact of the COVID-19 pandemic on renal transplantation in the UK. Clin Med (Lond). 2020;20(4):e82-6. doi: http://dx.doi.org/10.7861/ clinmed.2020-0183. PubMed.

9 Sabatino J, De Rosa S, Di Salvo G, Indolfi C. Impact of cardiovascular risk profile on COVID-19 outcome. A meta-analysis. PLoS One. 2020;15(8):e0237131. doi: http://dx.doi.org/10.1371/journal.pone.0237131. PubMed.

10 Tschopp J, L'Huillier AG, Mombelli M, Mueller NJ, Khanna N, Garzoni C, et al.; Swiss Transplant Cohort Study (STCS). First experience of SARS-CoV-2 infections in solid organ transplant recipients in the Swiss Transplant Cohort Study. Am J Transplant. 2020;20(10):2876-82. doi: http://dx.doi.org/10.1111/ajt.16062. PubMed.

11 Siddiqi HK, Mehra MR. COVID-19 illness in native and immunosuppressed states: A clinical-therapeutic staging proposal. J Heart Lung Transplant. 2020;39(5):405-7. doi: http://dx.doi.org/10.1016/ j.healun.2020.03.012. PubMed.
12 Breslow NE, Day NE. Statistical methods in cancer research. Volume II--The design and analysis of cohort studies. IARC Sci Publ. 1987;82(82):1-406. PubMed.

13 Federal Statistical Office. Population, 2nd Quarter 2020. [Online]. Accessed on: 2020 October 30. Available from: https://www.bfs.admin.ch/ bfs/en/home/statistics/population.assetdetail.14027789.html

14 The Principality of Liechtenstein. Country and people. [Online]. Accessed on: 2020 October 30. Available from: https://www.liechtenstein.li/en/country-and-people/society/population/

15 Federal Office of Public Health. Coronavirus: Situation in Switzerland. Epidemiological situation in Switzerland and the Principality of Liechtenstein. [Online]. Accessed on: 2020 October 30. Available from: https://www.bag.admin.ch/bag/en/home/krankheiten/ausbrueche-epidemien-pandemien/aktuelle-ausbrueche-epidemien/novel-cov/situationschweiz-und-international.html\#-1680104524

16 Boyarsky BJ, Massie AB, Love AD, Werbel WA, Durand CM, Avery RK, et al. Early experience with COVID-19 testing in transplantation. Transplant Direct. 2020;6(7):e572. doi: http://dx.doi.org/10.1097/ TXD.0000000000001024. PubMed.

17 Hernández-Galdamez DR, González-Block MA, Romo-Dueñas DK, Lima-Morales R, Hernández-Vicente IA, Lumbreras-Guzmán M, et al. Increased risk of hospitalization and death in patients with COVID-19 and pre-existing noncommunicable diseases and modifiable risk factors in Mexico. Arch Med Res. 2020;51(7):683-9. doi: http://dx.doi.org/ 10.1016/j.arcmed.2020.07.003. PubMed.

18 Bundesamt für Statistik. Schweizerische Gesundheitsbefragung 2017. BFS-Nummer 213-1708 (in German). [Online]. Accessed on: 2020 October 30. Available from: https://www.bfs.admin.ch/bfs/de/home/statistiken/gesundheit/erhebungen/sgb.assetdetail.9546738.html

19 Ikizler TA. COCID-19 and dialysis units: what do we know now and what should we do? A J. Am J Kidney Dis. 2020;76(1):1-3. doi: http://dx.doi.org/10.1053/j.ajkd.2020.03.008. PubMed.

20 Poletti P, Tirani M, Cereda D, Trentini F, Guzzetta G, Marziano V, et al Age-specific SARS-CoV-2 infection fatality ratio and associated risk factors, Italy, February to April 2020. Euro Surveill. 2020;25(31):2001383. doi: http://dx.doi.org/10.2807/ 1560-7917.ES.2020.25.31.2001383. PubMed.

21 Pormohammad A, Ghorbani S, Baradaran B, Khatami A, Turner RJ, Mansournia MA, et al. Clinical characteristics, laboratory findings, radiographic signs and outcomes of 61,742 patients with confirmed COVID-19 infection: A systematic review and meta-analysis. Microb Pathog. 2020;147:104390. doi: http://dx.doi.org/10.1016/j.micpath.2020.104390. PubMed.

22 Galvan NTN, Moreno NF, Garza JE, Bourgeois S, Hemmersbach-Miller M, Murthy B, et al. Donor and transplant candidate selection for solid organ transplantation during the COVID-19 pandemic. Am J Transplant. 2020;20(11):3113-22. doi: http://dx.doi.org/10.1111/ajt.16138. PubMed.

23 Kumar D, Manuel O, Natori Y, Egawa H, Grossi P, Han SH, et al. COVID-19: A global transplant perspective on successfully navigating a pandemic. Am J Transplant. 2020;20(7):1773-9. doi: http://dx.doi.org/ 10.1111/ajt.15876. PubMed.

24 Shah MB, Lynch RJ, El-Haddad H, Doby B, Brockmeier D, Goldberg DS. Utilization of deceased donors during a pandemic: argument against using SARS-CoV-2-positive donors. Am J Transplant. 2020;20(7):1795-9. doi: http://dx.doi.org/10.1111/ajt.15969. PubMed.

25 Kates OS, Fisher CE, Rakita RM, Reyes JD, Limaye AP. Emerging evidence to support not always "just saying no" to SARS-CoV-2 positive donors. Am J Transplant. 2020;20(11):3261-2. doi: http://dx.doi.org/ 10.1111/ajt.16119. PubMed.

26 Passamonti SM, Cannavò A, Trunzo V, Caporale V, Buonocore R, DeFeo TM. Solid organ transplantation in the coronavirus disease 2019 era: "the great bet" in the North Italy transplant program area. Transplant Proc. 2020;52(9):2631-6. doi: http://dx.doi.org/10.1016/j.transproceed.2020.07.001. PubMed. 